\title{
Studienpräferenzen von Gymnasiastinnen und Gymnasiasten: Wer entscheidet sich aus welchen Gründen für ein MINT-Studium?
}

\section{Belinda Aeschlimann, Walter Herzog und Elena Makarova}

Der Beitrag analysiert Studienpräferenzen von deutschschweizerischen Gymnasiastinnen und Gymnasiasten. Im Zentrum steht die Frage, aus welchen Gründen zukünftige Studierende ein Studium im Bereich der Mathematik, Informatik, Naturwissenschaft und Technik (MINT) (ab-)wählen. Dabei werden sowohl individuelle als auch kontextuelle Einflussfaktoren fokussiert. Die Ergebnisse zeigen, dass das Geschlecht der Jugendlichen sowie ihr Interesse und ihre Leistungen in den Fächern Mathematik, Physik und Chemie die grösste Vorhersagekraft für eine MINT-Studienwahl haben. Darüberhinaus fungieren intrinsische Studienwahlmotive der Gymnasiastinnen und Gymnasiasten als wesentliche Determinanten für die Wabl oder Abwahl eines MINT-Studiums.

\section{Einleitung}

Ein wiederkehrendes Ergebnis gleichstellungs- und bildungspolitischer Analysen der vergangenen Jahre ist die anhaltende Geschlechtersegregation bei der Berufsund Studienwahl in der Schweiz und in anderen europäischen Ländern (OECD, 2013; SKBF, 2014). Global betrachtet, gleicht sich das Bildungsniveau von Frauen und Männern zwar tendenziell an. Die Übertrittsquote vom Gymnasium an eine Hochschule liegt bei beiden Geschlechtern mittlerweile praktisch gleich hoch bei insgesamt 92 Prozent. Differenziert nach Hochschultyp, ist die Quote der Frauen, die an eine Universität übertreten, jedoch deutlich tiefer als diejenige der Männer. Frauen wählen häufiger tertiäre Lehrgänge an nicht-universitären Hochschulen, insbesondere in den Bereichen Lehrerbildung und Gesundheit (Bieri Buschor, Berweger, Kreck Frei \& Kappler, 2012; SKBF, 2014, S. 154). Auch innerhalb der universitären Studiengänge unterscheiden sich die Quoten nach dem Geschlecht der Studierenden. Während Studiengänge in Mathematik, Informatik, Naturwissenschaft und Technik (MINT) häufiger von Männern gewählt werden, entscheiden sich Frauen vorwiegend für ein Studium in einer Geistes- oder Sozialwissenschaft sowie in der Medizin. 
Dazu kommt, dass die Geistes- und Sozialwissenschaften von den Studierenden stark nachgefragt werden, während die Studiengänge mit naturwissenschaftlich-technischer Ausrichtung nicht nur mit Rekrutierungsproblemen zu kämpfen haben, sondern auch vergleichsweise hohe Abbruchquoten aufweisen (BFS, 2009, S. 5; Gehrig, Gardiol \& Schaerrer, 2010, S. 80; Larsen, Sommers \& Larsen, 2013, S. 23). Im internationalen Vergleich bewegt sich der Anteil der MINT-Abschlüsse in der Schweiz im unteren Mittelfeld, wobei das Verhältnis zwischen Frauen und Männern deutlich ungünstiger ausfällt als in anderen Ländern (SKBF, 2014). Eine Erhöhung der Attraktivität der MINT-Studiengänge und eine damit verbundene Steigerung des Frauenanteils könnten nicht nur dem prognostizierten Fachkräftemangel im Bereich der naturwissenschaftlich-technischen Berufe entgegenwirken, sondern auch die stereotype Polarisierung von Frauen- und Männerberufen entkräften (Leemann \& Keck, 2005).

Um die Attraktivität eines MINT-Studiums für junge Frauen und Männer zu erhöhen, ist es unabdingbar, die persönlichen und schulischen Determinanten einer MINT-Studienwahl aufzuklären. Der vorliegende Beitrag befasst sich daher mit der Frage, aus welchen individuellen und kontextuellen Gründen sich Gymnasiastinnen und Gymnasiasten für die Wahl einer MINT-Studienrichtung aussprechen. In Erweiterung der bisherigen Forschung werden Bedingungsfaktoren der MINT-Studienwahl an einer repräsentativen Stichprobe aus der deutschsprachigen Schweiz untersucht. Eine Neubeurteilung des Themas lohnt sich auch deshalb, da bildungspolitische Massnahmen sowie spezifische Programme (z.B. Mentoring) bisher relativ erfolglos blieben (Gilbert, 2009, S. 51).

\section{Forschungsstand}

Ein Studium bereitet zwar nicht unbedingt auf eine berufliche Tätigkeit vor, jedoch ist davon auszugehen, dass die Entscheidung für ein Studium eng mit der Wahl einer beruflichen Option verbunden ist, so dass die Studienwahl von Maturandinnen und Maturanden auch als Präferenz für ein bestimmtes Berufsfeld verstanden werden kann (Heublein \& Sommer, 2002, S. 10). Theorien der Berufs- und Studienwahl (für einen Überblick: Herzog, Neuenschwander \& Wannack, 2006) gehen davon aus, dass der Übergang von der Schule in den Beruf von mehreren interdependenten individuellen und kontextuellen Faktoren bestimmt wird (Eccles \& Wigfield, 2002). Der Spielraum, der den Jugendlichen bei der Entscheidungsfindung offen steht, wird wesentlich durch ihre soziale Herkunft, ihr Geschlecht und die erworbenen Kompetenzen beeinflusst (Brändle \& Grundmann, 2013, S. 58). «Die Auseinandersetzung mit primären und sekundären Herkunftseffekten bei Bildungsentscheidungen verweist auf die bildungssystemische Rahmung und sozialstrukturelle Verankerung von Berufswahlprozessen» (ebd., S. 70). Je nach Ansatz wird die Berufswahl aber auch in Abhängigkeit von institutionellen (z.B. Rahmenbedingungen zur Verein- 
barkeit von Familie und Beruf) oder sozialkonstruktivistischen Faktoren (z.B. soziale Konstruktion von Geschlecht) gesehen (Gildemeister, 2009). In unserer Studie werden insbesondere primäre Herkunftseffekte berücksichtigt. Während einige Studien bezüglich mathematisch-naturwissenschaftlicher Fächer belegen, dass Studierende eher aus einem nicht-akademischen Milieu stammen (Becker, Haunberger \& Schubert, 2010), finden andere Untersuchungen keinen oder nur einen geringen direkten Einfluss des Herkunftsmilieus auf die Wahl eines MINT-Studiums oder eines MINT-Berufs (Gehrig et al., 2010, S. 64; Lojewski, 2011, S. 334; Windolf, 1992, S. 93).

Psychologische Ansätze betrachten den Berufswahlprozess vorzugsweise im Hinblick auf die Passung zwischen Persönlichkeitsmerkmalen und den Charakteristika des anvisierten Berufsfeldes (Herzog et al., 2006). Angenommen wird, dass die Wahl eines Berufs durch Faktoren wie Fähigkeiten, Motive und Interessen beeinflusst wird. Dabei spielt auch das Geschlecht eine zentrale Rolle. Familiale und schulische Sozialisationsprozesse, die geschlechtsspezifische Rollenerwartungen und Werthaltungen vermitteln, prägen die Studienwahl von Frauen und Männern nachhaltig (Eccles, 2007; Gottfredson, 2002). So können mehrere Studien belegen, dass die Geschlechtstypik der Berufe ein zentrales Kriterium im Berufswahlprozess von Jugendlichen darstellt (Bubany \& Hansen, 2011; Buchmann \& Kriesi, 2012; Herzog et al., 2006).

Bei den Studienwahlmotiven wird häufig zwischen intrinsischen und extrinsischen Motiven unterschieden (z.B. Renn \& Pfennig, 2009). Als intrinsisch gelten Motive, die den Neigungen und Begabungen einer Person entsprechen. Besondere Aufmerksamkeit erfahren dabei oft soziale Motive. Als extrinsisch gelten Motive, bei denen äussere Faktoren wie ein hohes Einkommen oder Arbeitsmarktchancen für die Studienwahl ausschlaggebend sind.

Eine Reihe von Studien kann belegen, dass intrinsische Motive die Wahl eines Studiums stark beeinflussen (BFS, 2009; Briedis et al., 2008). Dabei spielt bei Frauen die intrinsische Motivation eine grössere Rolle als bei Männern, die sich bei der Berufs- bzw. Studienwahl stärker als extrinsisch motiviert erweisen (BFS, 2009, S. 7; Heine, Willich, Schneider \& Sommer, 2008, S. 137). Zudem sind Frauen im Vergleich zu Männern stärker sozial orientiert, was mit den von ihnen bevorzugten Studienrichtungen übereinstimmt (Bargel, 2007, S. 28).

Was die Studienrichtungen im MINT-Bereich anbelangt, so zeigt eine Analyse von Daten des Hochschul-Informations-Systems (HIS) in Deutschland, dass die Motivlage von Studierenden dieser Fächer im Vergleich zu derjenigen von Studierenden anderer Fachrichtungen stark extrinsisch ausgerichtet ist (Heine et al., 2008, S. 143). Damit übereinstimmend belegen andere Studien, dass soziale Motive für die Wahl einer mathematisch-naturwissenschaftlichen Studienrichtung eine untergeordnete Rolle spielen (Andreitz, Müller, Kramer \& Krainer, 2013, S. 8; Briedis et al., 2008, S. 33).

Neben intrinsischen und extrinsischen Motiven spielen bei der Studienwahl auch fachliche Interessen eine bedeutsame Rolle (Heine et al., 2008, S. 142ff.; 
Windolf, 1992). Empirisch erweist sich das Interesse am Studienfach sogar als wichtigste Determinante der Studienwahlentscheidung (z.B. BFS, 2009, S. 10). Das Fachinteresse von Schülerinnen und Schülern entwickelt sich im Laufe ihrer schulischen Karriere und wird von einer Reihe von Faktoren wie Fähigkeiten und Begabungen, aber auch durch die Unterrichtsgestaltung und durch ausserschulische Erfahrungen beeinflusst. Es bildet zudem nicht nur eine wesentliche Voraussetzung für das schulische Lernen (Köller, Baumert \& Schnabel, 2001), sondern korreliert in den naturwissenschaftlichen Fächern bei den Schülerinnen auch stärker mit der fachlichen Leistung als bei den Schülern (Blakemore, Berenbaum \& Liben, 2009, S. 94). Eine Analyse von TREE-Daten zeigt, dass zwischen der Mathematikleistung und dem Studienfachentscheid sowohl bei Männern als auch bei Frauen ein deutlicher Zusammenhang besteht (Gehrig et al., 2010, S. 67). Es scheint, dass die Mathematikleistung bei der Entscheidung für oder gegen ein MINT-Studium einen gewichtigen Faktor darstellt.

Die Fachleistung scheint insbesondere bei jungen Frauen für die Studienwahl bedeutsam zu sein, lassen sie sich doch durch eine ungünstige Note eher von einem Studium in dem betreffenden Fach abbringen als junge Männer (Bargel, 2007). Es ist jedoch davon auszugehen, dass die Geschlechtsunterschiede bei den Fachinteressen grösser sind als die Leistungsunterschiede, da sich gemäss PISA selbst Mädchen, die in Naturwissenschaften besonders kompetent sind, weniger für naturwissenschaftliche Fächer interessierten als Jungen (Prenzel, Schütte \& Walter, 2007). Darüberhinaus schätzen Mädchen ihre Leistungsfähigkeit in den MINT-Fächern generell tiefer ein als Jungen (Gehrig et al., 2010, S. 67).

Für unsere eigene Studie fokussieren wir drei Merkmalsbereiche, die wir aufgrund der referierten Befunde als bedeutsame Determinanten der Studienwahl einschätzen: strukturelle Faktoren (Geschlecht, sozioökonomische Stellung der Herkunftsfamilie), schulische Faktoren (fachliches Interesse, fachliche Leistung) und persönliche Faktoren (Studienwahlmotive).

\section{Methode}

\section{Untersuchungsdesign und Stichprobe}

Die zur Beantwortung der Forschungsfragen verwendeten Daten stammen aus dem Projekt "Geschlechtsuntypische Berufs- und Studienwahlen von jungen Frauen», das im Rahmen des Nationalen Forschungsprogramms 60 "Gleichstellung der Geschlechter» durchgeführt wurde. Insgesamt konnten bei 3032 Gymnasiastinnen und Gymnasiasten aus 16 Kantonen der Deutschschweiz mittels standardisierter schriftlicher Befragung im Klassenverband Daten erhoben werden. Für die Analysen der vorliegenden Studie werden die Daten von 2806 Jugendlichen (55.8\% weiblich; 44.2\% männlich) aus insgesamt 167 Klassen, die angegeben haben, unmittelbar nach erfolgreichem Abschluss des Gymnasiums ein Studium an einer Universität oder Fachhochschule aufzu- 
nehmen, verwendet. ${ }^{1}$ Die Jugendlichen befanden sich zum Zeitpunkt der Befragung (Frühjahr 2011) im letzten oder vorletzten Gymnasialjahr und waren durchschnittlich 19 Jahre $(S D=1.09)$ alt.

\section{Instrumente}

MINT-Studienwahl. Die Gymnasiastinnen und Gymnasiasten wurden gefragt, für welche Studienrichtung sie nach erfolgreich absolvierter Maturität optieren. Die abhängige Variable Studienwahl umfasst die Kategorien «MINT-Studienwahl und «Andere Studienwahl. Die Kategorie «MINT-Studienwahl`2 umfasst alle Studienrichtungen in den Bereichen Mathematik, Statistik, Informatik, Naturwissenschaften und Ingenieurwissenschaften, die überwiegend einen Frauenanteil unter 30 Prozent aufwiesen (BFS, 2012a, b). Die integrative Betrachtung der MINT-Fächer legt sich dadurch nahe, dass einerseits die deskriptive Analyse keine wesentlichen Unterschiede zwischen den Fachrichtungen ergeben hat, und andererseits die Anzahl weiblicher Fälle bei einzelnen Studienrichtungen zu gering ist, um multivariate Auswertungen fächerspezifisch durchzuführen. In den Analysen bildet daher die 〈MINT-Studienwahl〉 die Antwortkategorie 1 und die «Andere Studienwahl die Referenzkategorie 0.

Neben dem Geschlecht ( 1 = weiblich, 2 = männlich) wurden die folgenden unabhängigen Variablen verwendet: Sozioökonomischer Status (HISEI). Der sozioökonomische Status der Herkunftsfamilie wurde über die Angaben der Berufe der Eltern erfasst. Die Berufsangaben wurden mittels der International Standard Classification of Occupation ISCO 88 klassifiziert und anschliessend anhand des International Socio-Economic Index (ISEI) in eine metrische Skala transformiert. In die Analysen floss der HISEI (Highest ISEI) ein, der den höchsten Wert der Familie berücksichtigt ${ }^{3}$ (Min $=20, \operatorname{Max}=113, M=60.14$, $S D=15.51, \mathrm{ICC}=0.03)$. Zusätzlich wurde der Einfluss der Geschlechtstypik der Elternberufe überprüft, die jedoch keinen direkten statistisch signifikanten Erklärungsbeitrag zur MINT-Studienwahl von Gymnasiastinnen und Gymnasiasten liefern konnte. Wir gehen daher nicht weiter darauf ein.

Fachliches Interesse. Zur Erhebung des fachlichen Interesses wurden drei Fragen zum Interesse an den Fächern Mathematik, Physik und Chemie gestellt (SBFI, 2010, S. 5). Die Antworten wurden anschliessend zu einer Skala zusammengefasst ( $\mathrm{Min}=1, \operatorname{Max}=5, M=3.18, S D=.98, \alpha=.65, \mathrm{ICC}=0.11$ ).

Fachliche Leistung. Die fachliche Leistung wurde über die Erfragung der Zeugnisnoten in den Fächern Mathematik, Physik und Chemie erhoben (Note $1=$ schlechteste Note bis Note $6=$ beste Note). Auch hier wurden die Angaben $\mathrm{zu}$ einer Skala zusammengefasst $(\mathrm{Min}=1, \operatorname{Max}=9, M=6.23, S D=1.27$, $\alpha=.78$, ICC $=0.07$ ).

Studienwahlmotive. Zur Erhebung der Studienwahlmotive wurde ein Instrument von Renn und Pfenning (2009) verwendet. Auf der Basis von Faktorenanalysen konnten drei Subskalen mit je drei bis vier Items (Antwortvorgaben von $1=$ völlig unwichtig bis $5=$ sehr wichtig) gewonnen werden. Die Subskala 
Extrinsische Orientierung fasst Studienwahlmotive wie 'gute Aufstiegs- und Karrieremöglichkeiten` oder 'Verbesserung der Arbeitsmarktchancen〉 zusammen $(M=3.76, S D=.82, \alpha=.86, \mathrm{ICC}=0.04)$. Die Intrinsische Orientierung als weitere Subskala umfasst Studienwahlmotive wie eigenen Neigungen und Begabungen entsprechen oder eigene Verwirklichung) $(M=4.49, S D=.47$, $\alpha=.60$, ICC $=0.02)$. Bei der dritten Subskala Soziale Orientierung handelt es sich um Studienwahlmotive wie (mit familiären Pflichten vereinbaren` oder 'gesellschaftliche Zukunft mitgestalten` $(M=3.74, S D=.63, \alpha=.57$, ICC $=$ 0.03). Die internen Konsistenzen der Skalen sind in Anbetracht ihrer Kürze mit Cronbach's Alpha-Werten von .60 bis $.86^{4}$ akzeptabel.

\section{Statistische Analysen}

Die statistischen Analysen wurden mit dem Statistikprogramm SPSS 22.0 durchgeführt. Bei den deskriptiven Analysen wurden Mittelwertvergleiche ( $t$-Tests) und Chi-Quadrat-Tests durchgeführt. Für die explanative Analyse der Determinanten der MINT-Studienwahl wurde aufgrund der hierarchischen Datenstruktur ein generalisiertes lineares gemischtes Modell (GLMM) geschätzt, das die Mehrebenenstruktur der Daten bei der Parameterschätzung berücksichtigt. Fälle mit extremen Residuen wurden mittels Pearson Residual identifiziert und nach einer qualitativen Überprüfung aus den Berechnungen ausgeschlossen. Der Anteil fehlender Werte lag für die einzelnen Variablen unter 1 Prozent; einzig für die Skala der sozioökonomischen Stellung der Familie (HISEI) wurden aufgrund der hohen Anzahl fehlender Werte (16.6\%) Daten mittels Hilfsvariablen imputiert.

\section{Ergebnisse}

\section{Deskriptive Befunde}

Strukturelle Faktoren. Erwartungsgemäss ist der Anteil von Gymnasiastinnen und Gymnasiasten, die angeben, ein MINT-Studium aufnehmen zu wollen, deutlich geringer $(18.5 \%)$ als derjenige, der beabsichtigt, eine andere Fachrichtung zu belegen (81.5\%) (vgl. Tab. 1). Auffällig ist der signifikant tiefere Wert an MINT-Studienwahlen bei den Gymnasiastinnen (5.3\%) im Vergleich zu den Gymnasiasten (13.2\%). Dagegen sind die Gymnasiasten (31.0\%) im Vergleich zu den Gymnasiastinnen (50.5\%) in allen anderen Studienrichtungen signifikant untervertreten.

Neben dem Geschlecht erweist sich auch der sozioökonomische Status der Herkunftsfamilie als bedeutungsvolle Determinante der Studienwahl der Gymnasiastinnen und Gymnasiasten (vgl. Tab. 2). Der HISEI der potentiellen MINT-Studentinnen ist im Vergleich mit den potentiellen Studentinnen anderer Fachrichtungen signifikant höher. Bei den Gymnasiasten lässt sich kein analoger Effekt nachweisen. 
Tabelle 1: Geschlechtstypik der Studienwahl

\begin{tabular}{llccc}
\hline & \multicolumn{2}{c}{ Geschlecht } & \\
& & weiblich & männlich & Alle \\
\hline \multirow{2}{*}{ MINT-Studienrichtungen } & & $N=149$ & $N=369$ & $N=518$ \\
& Within Geschlecht & $9.5 \%$ & $29.8 \%$ & \\
& Total & $5.3 \%$ & $13.2 \%$ & $18.5 \%$ \\
& & $\mathrm{R}_{\mathrm{adj}}=-13.7$ & $\mathrm{R}_{\mathrm{adj}}=13.7$ & \\
\hline Andere Studienrichtungen & \multirow{2}{*}{ Within Geschlecht } & $N=1413$ & $N=869$ & $N=2282$ \\
& Total & $50.5 \%$ & $70.2 \%$ & \\
& & $\mathrm{R}_{\mathrm{adj}}=13.7$ & $\mathrm{R}_{\mathrm{adj}}=-13.7$ & \\
& & &
\end{tabular}

Anmerkungen. Chi-Quadrat-Test $(1,2800)=188.15, p<.001$, Adjusted Residual;

$\mathrm{R}_{\mathrm{adj}} \geq \pm 3.3=p<.001$.

Tabelle 2: Strukturelle, schulische und persönliche Faktoren nach Studienwahl und Geschlecht

\begin{tabular}{|c|c|c|c|c|}
\hline & $\begin{array}{c}\text { MINT-Stu- } \\
\text { dienwahl } \\
M(S D)\end{array}$ & $\begin{array}{c}\text { Andere } \\
\text { Studienwahl } \\
M(S D)\end{array}$ & $\begin{array}{c}\text { Studienwahl } \\
\text { Differenz } \\
p(\mathrm{~d})\end{array}$ & $\begin{array}{c}\text { Geschlechter } \\
\text { Differenz } \\
p(\mathrm{~d})\end{array}$ \\
\hline \multicolumn{5}{|c|}{ Strukturelle Faktoren } \\
\hline \multicolumn{5}{|c|}{ HISEI } \\
\hline W & $62.26(14.00)$ & $58.58(15.67)$ & ** $(-.26)$ & \multirow{2}{*}{$\begin{array}{l}\text { AS: } \mathrm{m}>\mathrm{w}, \\
* * *(.19)\end{array}$} \\
\hline M & $62.12(15.41)$ & $61.50(15.30)$ & n.s. & \\
\hline \multicolumn{5}{|c|}{$\begin{array}{l}\text { Schulische Faktoren } \\
\text { Fachinteresse }\end{array}$} \\
\hline W & $3.88(.65)$ & $2.94(.91)$ & $* * *(-.95)$ & \multirow{2}{*}{$\begin{array}{c}\text { AS: } \mathrm{m}>\mathrm{w}, \\
* * *(.19)\end{array}$} \\
\hline M & $3.99(.73)$ & $3.12(.98)$ & $* * *(-94)$ & \\
\hline \multicolumn{5}{|c|}{ Fachleistung } \\
\hline W & $8.82(1.19)$ & $8.05(1.26)$ & $* * *(-.57)$ & \multirow[t]{2}{*}{ n.s. } \\
\hline M & $9.00(1.13)$ & $8.08(1.21)$ & $* * *(-.76)$ & \\
\hline \multicolumn{5}{|c|}{ Persönliche Faktoren } \\
\hline \multicolumn{5}{|c|}{ Extrinsische Orientierung } \\
\hline W & $3.66(.77)$ & $3.67(.77)$ & n.s. & \multirow{2}{*}{$\begin{array}{c}\text { MINT/AS: } \\
\mathrm{m}>\mathrm{w}, \\
* *(.29) / \\
{ }^{* * *}(.27)\end{array}$} \\
\hline M & $3.89(.79)$ & $3.89(.89)$ & n.s. & \\
\hline \multicolumn{5}{|c|}{ Intrinsische Orientierung } \\
\hline W & $4.46(.51)$ & $4.53(.42)$ & n.s. & \multirow{2}{*}{$\begin{array}{l}\text { AS: } \mathrm{w}>\mathrm{m}, \\
* * *(-.23)\end{array}$} \\
\hline M & $4.52(.49)$ & $4.42(.54)$ & ** $(-.19)$ & \\
\hline \multicolumn{5}{|c|}{ Soziale Orientierung } \\
\hline W & $3.61(.58)$ & $3.85(.56)$ & $* * *(.35)$ & \multirow{2}{*}{$\begin{array}{c}\mathrm{AS}: \mathrm{w}>\mathrm{m}, \\
* * *(-.35)\end{array}$} \\
\hline M & $3.60(.71)$ & $3.64(.66)$ & n.s. & \\
\hline
\end{tabular}

Anmerkungen. Mittelwertvergleiche mittels $t$-Tests für unabhängige Stichproben; $M=$ Mittelwert, $S D=$ Standardabweichung; n.s. $>.05,{ }^{* *} p<.01,{ }^{* * *} p<.001, \mathrm{~d}=$ Effektstärke; MINT = MINT-Studienwahl, AS = Andere Studienwahl, $\mathrm{W}=$ weiblich, $\mathrm{M}=$ männlich. 
Persönliche Faktoren. Die befragten Jugendlichen nennen unabhängig vom Geschlecht in erster Linie intrinsische Motive für die Wahl ihres Studiums. Die intrinsische Motivlage ist jedoch bei den männlichen Jugendlichen mit MINT-Studienwahl deutlich ausgeprägter als bei ihren Geschlechtsgenossen, die ein anderes Fach in Erwägung ziehen. Bei den weiblichen Jugendlichen gibt es keinen Unterschied im Ausmass an intrinsischer Motivation in Bezug auf die Studienfachrichtung. Extrinsische Motive werden von den potentiellen MINT-Studierenden häufiger genannt als von den potentiellen Studierenden anderer Studienrichtungen. Darüber hinaus weicht das Ausmass an extrinsischer Motivation bei Gymnasiastinnen mit einer MINT-Studienwahl nicht signifikant vom Ausmass an extrinsischer Motivation bei Gymnasiastinnen mit anderer Studienwahl ab. Ebenfalls keinen signifikanten Unterschied in der extrinsischen Motivation gibt es bei Gymnasiasten, die sich für ein MINT-Studium entschieden haben, verglichen mit Gymnasiasten mit anderer Studienwahl. Festzuhalten bleibt indes, dass Gymnasiasten bezüglich der Wahl ihres Studiums generell stärker extrinsisch motiviert sind als Gymnasiastinnen.

Erwartete geschlechtsspezifische Unterschiede finden sich bei den sozialen Motiven. Gymnasiastinnen mit anderer Studienwahl gewichten soziale Motive signifikant stärker als Gymnasiastinnen mit MINT-Studienwahl. Bei den Gymnasiasten ist der Stellenwert sozialer Motive generell tief. Werden darüberhinaus die Geschlechtsunterschiede betrachtet, so weisen die Gymnasiastinnen mit anderer Studienwahl signifikant höhere Werte in der sozialen Orientierung auf, während in der Gruppe der MINT-Wählenden kein signifikanter Unterschied zwischen den Geschlechtern festzustellen ist.

\section{Determinanten der MINT-Studienwahl}

Um über die deskriptiven Befunde hinaus nach Determinanten der Wahl eines MINT-Studiums zu suchen, wurden im Rahmen eines GLMM insgesamt drei Modelle geschätzt. Das 0-Modell (ohne Prädiktoren) zeigte zunächst, dass die Zugehörigkeit zu einer Klasse eine Mehrebenenmodellierung erforderlich macht $(p<.001)$. Das Erfordernis bestätigte sich in der Überprüfung der ICCs der Prädiktoren, die Varianzanteile zwischen 3 und 11 Prozent auf die Klassenzugehörigkeit zurückführen. Das Modell 1 beinhaltet alle vorgestellten z-standardisierten Prädiktoren und deren Interaktionen mit dem Geschlecht. Nahezu alle Prädiktoren erweisen sich als voraussagekräftig. Einzig der HISEI und die extrinsische Orientierung sowie deren Interaktion mit dem Geschlecht müssen aufgrund fehlender Voraussagekraft aus dem anschliessend geschätzten Modell 2 ausgeschlossen werden, was verglichen mit dem AIC-Koeffizienten zu einer besseren Modellanpassung führt. Modell 2 umfasst schliesslich neben dem Geschlecht die Prädiktoren Fachinteresse, Fachleistung, intrinsische Orientierung und soziale Orientierung, die alle in einem Schritt in das Modell aufgenommen wurden. Zusätzlich wurden im selben Schritt die Interaktionen mit den entsprechenden Prädiktoren geschätzt. Über die genannten Prädiktoren hinaus 
erweisen sich auch die Interaktionen des Geschlechts mit dem Fachinteresse, der Fachleistung und der intrinsischen Orientierung als statistisch signifikant (mindestens $p<.05)$. Im Folgenden wird das Modell anhand der Effekt-Koeffizienten $\operatorname{Exp}(\beta)$ erläutert (vgl. Tab. 3).

\begin{tabular}{|c|c|c|c|c|c|}
\hline Modellterm & $\beta$ & $S E$ & $t$ & Sig. & $\operatorname{Exp}(\beta)$ \\
\hline (Intercept) & -1.44 & 0.10 & -14.05 & .000 & 0.24 \\
\hline \multicolumn{6}{|l|}{ Strukturelle Faktoren } \\
\hline $\mathrm{W}$ & -1.21 & 0.15 & -7.89 & .000 & 0.30 \\
\hline $\mathrm{M}$ & $0^{\mathrm{a}}$ & & & & \\
\hline \multicolumn{6}{|l|}{ Schulische Faktoren } \\
\hline Fachinteresse (FI) & .89 & .10 & 8.77 & .000 & 2.44 \\
\hline $\mathrm{W}^{*} \mathrm{FI}$ & .41 & .18 & 2.31 & .021 & 1.50 \\
\hline $\mathrm{M}^{*} \mathrm{FI}$ & $0^{\mathrm{a}}$ & & & & \\
\hline Fachleistung (FL) & .42 & .09 & 4.56 & .000 & 1.52 \\
\hline $\mathrm{W}^{*} \mathrm{FL}$ & -.40 & .15 & -2.73 & .006 & .67 \\
\hline $\mathrm{M}^{*} \mathrm{FL}$ & $0^{\mathrm{a}}$ & & & & \\
\hline \multicolumn{6}{|l|}{ Persönliche Faktoren } \\
\hline Intrinsische Orientierung (IO) & .18 & .08 & 2.45 & .014 & 1.20 \\
\hline $\mathrm{W}^{*} \mathrm{IO}$ & -.30 & .13 & -2.30 & .022 & .74 \\
\hline $\mathrm{M}^{*} \mathrm{IO}$ & $0^{\mathrm{a}}$ & & & & \\
\hline Soziale Orientierung (SO) & -.23 & .07 & -3.24 & .001 & .79 \\
\hline $\mathrm{W} * \mathrm{SO}$ & -.24 & .13 & -1.83 & .068 & .79 \\
\hline $\mathrm{M} * \mathrm{SO}$ & $0^{\mathrm{a}}$ & & & & \\
\hline
\end{tabular}

Anmerkungen. Abhängige Variable: MINT-Studienwahl (Referenzkategorie = Andere Studienwahl); $\beta=$ Regressionskoeffizient, $S E=$ Standardfehler; a Auf O gesetzt, da dieser Parameter redundant ist; $W=$ weiblich, $M=$ männlich .

Die Wahrscheinlichkeit, sich für ein MINT-Studium zu entscheiden, ist für Gymnasiastinnen im Vergleich zu Gymnasiasten deutlich reduziert $(\operatorname{Exp}(\beta)=$ .30). Gymnasiasten haben eine rund 70 Prozent grössere Chance, sich für ein MINT-Studium zu entscheiden, als Gymnasiastinnen. Die grösste Voraussagekraft für eine MINT-Studienwahl liefern jedoch die schulischen Faktoren. Dabei erweist sich das Fachinteresse in Mathematik, Physik und Chemie als bedeutsamster Prädiktor. Die Wahrscheinlichkeit für Gymnasiastinnen und Gymnasiasten, ein MINT-Studium zu ergreifen, steigt um das 2.4fache an $(\operatorname{Exp}(\beta)=2.44)$, wenn sie sich für die Fächer Mathematik, Physik und Chemie interessieren. Bei gleich hohem Fachinteresse wie die Gymnasiasten, wählen die Gymnasiastinnen zudem mit einer 50 Prozent höheren Wahrscheinlichkeit ein MINT-Studium als ihre männlichen Mitschüler $(\operatorname{Exp}(\beta)=1.50)$. Eine hohe Fachleistung in den Fächern Mathematik, Physik und Chemie geht schliesslich bei beiden Geschlechtern mit einer rund um die Hälfte $(\operatorname{Exp}(\beta)=1.52)$ erhöhten Wahrscheinlichkeit einer MINT-Studienwahl einher. Jedoch wählen Gymnasi- 
astinnen mit gleich hohen Leistungen in den besagten Fächern deutlich weniger oft $(\operatorname{Exp}(\beta)=.67)$ ein Studium in einem MINT-Fach als ihre männlichen Mitschüler.

Unter den Studienwahlmotiven erweist sich insbesondere die soziale Orientierung als bedeutsam. Soziale Motive verringern unabhängig vom Geschlecht die Wahrscheinlichkeit, dass ein MINT-Studium gewählt wird, um 21 Prozent $(\operatorname{Exp}(\beta)=.79)$. Intrinsische Motive hingegen erhöhen die Wahrscheinlichkeit, dass die Studienwahl auf ein MINT-Fach fällt, um 21 Prozent $(\operatorname{Exp}(\beta)=.79)$. Darüberhinaus ziehen Gymnasiastinnen jedoch um rund einen Viertel weniger häufig ein MINT-Studium in Betracht verglichen mit Gymnasiasten mit gleich stark ausgeprägter intrinsischer Studienmotivation $(\operatorname{Exp}(\beta)=.74)$.

\section{Diskussion}

Ziel der vorliegenden Studie war die Aufklärung von Determinanten der MINT-Studienwahl bei jungen Frauen und Männern, die kurz vor Abschluss des Gymnasiums stehen. Die Ergebnisse zeigen, dass die Bereitschaft, ein MINT-Studium an einer Universität oder Fachhochschule aufzunehmen, bei Deutschschweizer Gymnasiastinnen und Gymnasiasten vergleichsweise gering ist und gerade einmal bei 18.5 Prozent liegt. Wie in anderen Studien, zeigt sich aber auch in unserer Studie, dass vor allem junge Frauen wenig Neigung zeigen, ein Studium in einem MINT-Fach aufzunehmen (BFS, 2009, S. 5; Gehrig et al., 2010, S. 77).

Obwohl sich das Geschlecht als gewichtige Determinante einer MINT-Studienwahl erweist, sind andere Einflussfaktoren ebenso bedeutsam und vermögen den Geschlechtereffekt teilweise zu neutralisieren. So zeigen die deskriptiven Befunde unserer Studie, dass sich potentielle Studierende eines MINT-Faches in mehrfacher Hinsicht von potentiellen Studierenden anderer Studienfächer unterscheiden.

Erstens sind Gymnasiastinnen aus sozioökonomisch besser gestellten Familien eher bereit, ein MINT-Studium aufzunehmen, als deren Geschlechtsgenossinnen aus sozioökonomisch schlechter situierten Herkunftsfamilien. Möglicherweise ist der familiäre Rückhalt für eine geschlechtsuntypische Studienwahl bei besser gestellten Gymnasiastinnen grösser, oder ihre Bereitschaft zu normabweichenden Entscheidungen wird von den Eltern eher gestützt. Für beide Interpretationen, die sich nicht ausschliessen müssen, könnte sprechen, dass sich bei der Studienwahl der Gymnasiasten keine Abhängigkeit vom sozioökonomischen Status der Familie findet.

Zweitens ist die extrinsische motivationale Orientierung in Bezug auf die unterschiedlichen Studienwahlen der Geschlechter nicht unterscheidend. Jedoch weisen die jungen Männer generell eine höhere extrinsische Studienwahlmotivation auf als die jungen Frauen. Dies bestätigt Ergebnisse bisheriger 
Untersuchungen, die Männern bei der Berufs- bzw. Studienwahl eine stärkere extrinsische Motivation attestieren (BFS, 2009, S. 7; Lojewski, 2011, S. 339). Damit übereinstimmend spielt drittens die intrinsische Motivation bei der Studienwahl sowohl bei den Gymnasiastinnen wie bei den Gymnasiasten eine grössere Rolle als die extrinsische Motivation. Auch damit werden bisherige Forschungsbefunde bestätigt (Bargel et al., 2008; Lojewski, 2011). Der Befund gilt insbesondere für die Wahl eines MINT-Studiums, wo sich bei der intrinsischen Motivation keine Geschlechterdifferenz zeigt, während bei der Wahl eines anderen Studiums die intrinsische Orientierung bei den Frauen stärker ausgeprägt ist als bei den Männern.

Viertens entscheiden sich Gymnasiastinnen mit einer starken sozialen Orientierung eher nicht für ein MINT-Studium. Tun sie es trotzdem, zeigt sich im Ausmass an sozialer Orientierung, das auf einem vergleichsweise tiefen Niveau liegt, keine Differenz zwischen den Geschlechtern. Es bestätigen sich damit Befunde auch für den deutschschweizerischen Kontext, die naturwissenschaftlich-technische Berufe mit einer eher geringen sozialen Orientierung in Verbindung bringen (u.a. Blakemore et al., 2009; Eccles, 2007).

Fünftens verweisen die Ergebnisse unserer Studie auf die zentrale Bedeutung schulischer Determinanten für eine MINT-Studienwahl, wobei sich das fachliche Interesse als besonders bedeutsame Einflussgrösse erweist, und zwar unabhängig vom Geschlecht. Aber auch die fachliche Leistung stellt einen einflussreichen Faktor dar, der die Wahl eines MINT-Studiums - wiederum unabhängig vom Geschlecht - massgeblich bestimmt.

Die Zusammenführung der deskriptiven Ergebnisse in einem explanativen Modell bestätigt im Wesentlichen die Determinanten der MINT-Studienwahl bei schweizerischen Gymnasiastinnen und Gymnasiasten. Die vergleichende Analyse bei weiblichen und männlichen Jugendlichen im Rahmen eines integrativen Modells erlaubt es, über die in der Geschlechterforschung oft wenig fruchtbare Beschränkung auf eines der beiden Geschlechter hinauszugehen. Ein gemeinsames Erklärungsmodell erweist sich vor allem aus pädagogischer Sicht als vorteilhaft, findet doch der gymnasiale Unterricht in den mathematisch-naturwissenschaftlichen Fächern im Normalfall koedukativ statt.

Unser Erklärungsmodell bestärkt insbesondere die Bedeutsamkeit der Faktoren fachliche Leistung und fachliches Interesse in Mathematik, Physik und Chemie als Determinanten einer MINT-Studienwahl. Dass die Leistung in Mathematik, Physik und Chemie von starkem Einfluss auf die Wahl eines MINT-Studiums ist, mag trivial erscheinen. Umso bedeutsamer ist, dass sich auch bei deutschschweizerischen Gymnasiastinnen nicht die Leistung, sondern das Fachinteresse als ausschlaggebend für die Wahl eines MINT-Studiums erweist. Bei gleicher Leistung wie ihre männlichen Peers ist ihre Bereitschaft, ein MINT-Studium aufzunehmen, um 33 Prozent reduziert, ein Ergebnis, das mit anderen Untersuchungen kompatibel ist (Eccles \& Wigfield, 2002; Taskinen, Asseburg \& Walter, 2009). Vermutlich ist die grössere Bereitschaft der Mädchen, 
sich den Leistungsnormen der Schule zu unterwerfen, dafür verantwortlich, dass sie auch dann gute Noten erbringen, wenn ihr Interesse an einem Fach gering ist (Duckworth \& Seligman, 2005).

Wie wichtig die motivationalen Faktoren für die Wahl eines MINTStudiums insbesondere bei jungen Frauen sind, zeigen auch die intrinsische und die soziale Orientierung, die bei starker Ausprägung die Bereitschaft zu einem MINT-Studium reduzieren. Angelehnt an das Erwartungs-Wert-Modell von Eccles, das auf schulische Leistungen und berufliche Entscheidungen ausgerichtet ist (Eccles \& Wigfield, 2002), könnte in einer an den Schülerinnen orientierten interessenfördernden Unterrichtsgestaltung ein nicht ausgeschöpftes Potential liegen, um die motivationale Defizite der Schülerinnen in Fächern wie Mathematik, Physik und Chemie aufzufangen (Aeschlimann, Herzog \& Makarova, 2014).

Abschliessend ist auf einige Beschränkungen unserer Studie hinzuweisen. So konnten wir lediglich die intendierte, nicht aber die faktische Studienwahl erheben. In weiterführenden Analysen gälte es, die vorliegenden Ergebnisse in Rahmen längsschnittlicher Studien zu validieren. Zudem wäre es wünschenswert, die MINT-Fächer, aber auch die anderen Studienrichtungen, intern stärker zu differenzieren. In Rechnung zu stellen ist zudem, dass die Studienwahl bei beiden Geschlechtern multipel determiniert ist (Gottfredson, 2002). Die von uns untersuchten Determinanten sind daher zweifellos nicht erschöpfend. Jedoch erlauben sie, pädagogische Massnahmen zur Erhöhung des Anteils von Studierenden in MINT-Fächern vorzuschlagen, die pragmatisch begründet sind und daher eher Aussicht auf Akzeptanz in der Schulpraxis bieten als ein «vollständiges» Erklärungsmodell.

\section{Anmerkungen}

1 Die Überrepräsentation des weiblichen Geschlechts ist einerseits durch den Sachverhalt bedingt, dass eine Mehrzahl von männlichen Befragten angab, nach erfolgreichem Abschluss des Gymnasiums eine praktische Tätigkeit ohne weitere Ausbildung aufzunehmen, andererseits eine Folge der aktuellen Überrepräsentation des weiblichen Geschlechts in den gymnasialen Bildungsgängen (BFS, 2014).

2 In unserer Studie sind die universitären Studienrichtungen Mathematik, Statistik, Informatik, Naturwissenschaften (exkl. Medizin und Pharmazie, inkl. Biologie und Geographie) und Ingenieurwissenschaften wie Bau-, Elektro-, Maschinen-, Werkstoffingenieurwissenschaften darin enthalten. In den nicht-universitären Studienrichtungen sind darin die Bereiche Technik/ Ingenieurwissenschaften und IT/ Computer erfasst.

3 Die Berücksichtigung des jeweils höheren Berufsstatus (Klassenlage) hat den Vorteil, dass der starken Zunahme der Frauenerwerbstätigkeit und dem Wandel in den Familienbildungs- und -aufösungsprozessen Rechnung getragen werden kann (z.B. Hadjar \& Berger, 2010).

4 Werden die Skalen nach der Vorhersageformel nach Spearman-Brown (Lienert \& Raatz, 1994, S. 210) auf 12 Items hochtransponiert erreichen alle drei Skalen Alpha-Wert zwischen .83 und .96, was auf eine gute bis sehr gute interne Konsistenz der Skalen hinweist. 


\section{Literatur}

Aeschlimann, B., Herzog, W. \& Makarova, E. (2014). Frauen in MINT-Berufen: Retrospektive Wahrnehmung des mathematisch-naturwissenschaftlichen Unterrichts auf der Sekundarstufe I. Zeitschrift für Bildungsforschung. DOI 10.1007/s35834-014-0111-y.

Andreitz, I., Müller, F. H., Kramer, D. \& Krainer, K. (2013). Wer studiert Technik? Eine Befragung österreichischer SchülerInnen und Studierender. Wissenschaftlicher Beitrag Nr. 7. Klagenfurt: Alpen-Adria-Universität.

Bargel, T. (2007). Soziale Ungleichheit im Hochschulwesen. Barrieren für Bildungsaufsteiger. Hefte zur Bildungs- und Hochschulforschung, Arbeitsgruppe Hochschulforschung (Heft 49), Universität Konstanz.

Bargel, T., Ramm, M. \& Multrus, F. (2008). Studiensituation und studentische Orientierungen. 10. Studierendensurvey an Universitäten und Fachhochschulen. Bonn: BMBF.

Becker, R., Haunberger, S. \& Schubert, F. (2010). Studienfachwahl als Spezialfall der Ausbildungsentscheidung und Berufswahl. Zeitschrift für Arbeitsmarktforschung, 42(4), 292-310.

BFS (2009). Studienfachwahl und Hochschulwahl. Motivationale Aspekte. Neuchâtel: Bundesamt für Statistik.

BFS (2012a). Abschlüsse der Fachhochschulen (inkl. PH): Basistabellen. Neuchâtel: Bundesamt für Statistik.

BFS (2012b). Abschlüsse der universitären Hochschulen: Basistabellen. Neuchâtel: Bundesamt für Statistik.

BFS (2014). Maturitätsquote nach Maturitätstyp und Geschlecht. Neuchâtel: Bundesamt für Statistik.

Bieri Buschor, C., Berweger, S., Keck Frei, A. \& Kappler, C. (2012). "Geschlechts(un-)typische” Studienwahl. Projektbericht. Zürich: Pädagogische Hochschule.

Blakemore, J. E. O., Berenbaum, S. A. \& Liben, L. S. (2009). Gender development. New York: Psychology Press.

Brändle, T. \& Grundmann, M. (2013). Soziale Determinanten der Studien und Berufswahl: theoretische Konzepte und empirische Befunde. In T. Brüggemann \& S. Rahn (Hrsg.), Berufsorientierung (S. 58-72). Münster: Waxmann.

Briedis, K., Egorova, T., Heublein, U., Lörz, M., Middendorff, E., Quast, H. \& Spangenberg, H. (2008). Studienaufnahme, Studium und Berufsverbleib von Mathematikern. Hannover: HIS.

Bubany, S. T. \& Hansen, J.-I. C. (2011). Birth cohort change in the vocational interests of female and male college students. Journal of Vocational Behavior, 78, 59-67.

Buchmann, M. \& Kriesi, I. (2012). Geschlechtstypische Berufswahl: Begabungszuschreibungen, Aspirationen und Institutionen. Kölner Zeitschrift für Soziologie und Sozialpsychologie, 52, 256-280.

Duckworth, A. L. \& Seligman, M. E. P. (2005). Self-Discipline Outdoes IQ in Predicting Academic Performance of Adolescents. Psychological Science, 16, 939-944.

Eccles, J. S. (2007). Where are all the women? Gender differences in participation in physical science and engineering. In S. J. Ceci \& W. M. Williams (Eds.), Why aren't more woman in science? Top researchers debate the evidence (pp. 199-210). Washington, DC: American Psychological Association.

Eccles, J. S. \& Wigfield, A. (2002). Motivational beliefs, values, and goals. Annual Review of Psychology, 53, 109-132.

Gehrig, M., Gardiol, L. \& Schaerrer, M. (2010). Der MINT-Fachkräftemangel in der Schweiz. Bern: SBF.

Gilbert, A.-F. (2009). Gender and disciplinary cultures: Comparing mechanical engineering and materials science. Equal Opportunities International, 28, 24-35.

Gildemeister, R. (2009). Soziale Konstruktion von Geschlecht: Theorieangebote und offene Fragen. In Enzyklopädie Erziehungswissenschaft Online. 
Gottfredson, L. S. (2002). Gottfredson's theory of circumscription, compromise, and self-creation. In D. Brown (Ed.), Career Choice and Development (4th Ed.) (pp. 85-148). San Francisco: Jossey-Bass.

Hadjar, A. \& Berger, J. (2010). Dauerhafte Bildungsungleichheiten in Westdeutschland, Ostdeutschland und der Schweiz. Zeitschrift für Soziologie, 39, 182-201.

Heine, C. Willich, J., Schneider, H. \& Sommer, D. (2008). Studienanfänger im Wintersemester 2007/08. Wege zum Studium, Studien- und Hochschulwahl, Situation bei Studienbeginn. Hannover: HIS.

Herzog, W. (1998). Chancengleichheit und naturwissenschaftliche Bildung. Zur Förderung von Mädchen im koedukativen Physikunterricht. In E. Nadai \& T.-H. Ballmer-Cao (Hrsg.), Grenzverschiebungen. Zum Wandel des Geschlechterverhältnisses in der Schweiz (S. 119-146). Zürich: Rüegger.

Herzog, W., Neuenschwander, M. P. \& Wannack, E. (2006). Berufswahlprozess. Wie sich Jugendliche auf ihren Beruf vorbereiten. Bern: Haupt.

Heublein, U. \& Sommer, D. (2002). Studienanfänger 2000/2001: Fachinteresse und berufliche Möglichkeiten bestimmen die Studienfachwahl. Hannover: HIS-Kurzinformationen A2/2002.

Köller, O., Schnabel, K. \& Baumert, J. (2001). Does interest matter? The relationship between academic interest and achievement in mathematics. Journal for Research in Mathematics Education, 32, 448-470.

Larsen, M. R., Sommersel, H. B. \& Larsen, M. S. (2013). Evidence on Drop-out Phenomena at Universities. Systematic Review. Copenhagen: Danish Clearinghouse for Educational Research.

Leemann, R. J. \& Keck, A. (2005). Der Übergang von der Ausbildung in den Beruf. Die Bedeutung von Qualifikation, Generation und Geschlecht. Neuchâtel: BFS.

Lienert, G. A. \& Raatz, U. (1994). Testaufbau und Testanalyse. Weinheim: Psychologie Verlags Union.

Lojewski, J. (2011). Geschlecht und Studienfachwahl - fachspezifischer Habitus oder geschlechtsspezifische Fachkulturen? In P. Bornkessel \& J. Asdonk (Hrsg.), Der Übergang Schule - Hochschule (S. 279-348). Wiesbaden: VS.

OECD (2013). Gleichstellung der Geschlechter. Zeit zu Handeln. OECD Publishing. Zugriff am 03.06.2014 unter http://dx.doi.org/10.1787/9789264190344-de

Prenzel, M., Schütte, K. \& Walter, O. (2007). Interesse an den Naturwissenschaften. In M. Prenzel, C. Artelt, J. Baumert, W. Blum, M. Hammann, E. Klieme \& R. Pekrun (Hrsg.), PISA 2006. Die Ergebnisse der dritten internationalen Vergleichsstudie (S. 107-124). Münster: Waxmann.

Renn, O. \& Pfenning, U. (2009). Nachwuchsbarometer Technikwissenschaften. Fragebogen. Stuttgart: Universität Stuttgart.

SBFI (2010). Evaluation der Schweizerischen Maturitätsreform. Fragebogen der Befragung der Abschlussklassen. Bern: Staatssekretariat für Bildung, Forschung und Innovation.

SKBF (2014). Bildungsbericht Schweiz 2014. Aarau: Schweizerische Koordinationsstelle für Bildungsforschung.

Taskinen, P., Asseburg, R. \& Walter, O. (2009). Wer möchte später einen naturwissenschaftsbezogenen oder technischen Beruf ergreifen? In M. Prenzel \& J. Baumert (Hrsg.), Vertiefende Analysen zu PISA 2006 (S. 79-105). Wiesbaden: VS.

Windolf, P. (1992). Fachkultur und Studienfachwahl: Ergebnisse einer Umfrage unter Studienanfängern. Kölner Zeitschrift für Soziologie und Sozialpsychologie, 44(1), S. 76-98.

Schlagworte: Gymnasium, Studienwahl, MINT, Geschlecht, Studienwahlmotive, Fachspezifische Interessen 


\section{Préférences d'études de lycéens et lycéennes: qui décide de suivre un cursus MINT et pour quelles raisons?}

\section{Résumé}

Cet article analyse les préférences de sélection d'études de lycéens et lycéennes de Suisse allemande, en se concentrant sur les motifs qui poussent les futurs étudiants à choisir ou à ne pas choisir un cursus en mathématiques, en informatique, en sciences naturelles ou en technique (MINT). L'analyse prend également en compte des facteurs d'influence individuels et contextuels. Les résultats démontrent que le sexe, un intérêt marqué pour des matières mathématiques et scientifiques de même que les compétences existantes dans ces domaines constituent les facteurs principaux d'un choix pour un cursus MINT. De plus, dans ce processus de choix d'études, des raisons de sélection intrinsèques jouent aussi un rôle déterminant en faveur ou au détriment d'une formation dans une branche MINT.

Mots-clés: Lycée, choix d'études - MINT, sexe, motifs de choix d'une branche d'études, intérêts spécifiques pour la branche

\section{Preferenze curricolari di alunni e alunne del ginnasio: chi e per quali ragioni sceglie un corso di studi MINT?}

\section{Riassunto}

L'articolo analizza le preferenze nella scelta degli studi di alunne e alunni ginnasiali della Svizzera tedesca. L'articolo sostanzialmente s'interroga su quali motivi spingano i futuri studenti a scegliere (o a scartare) un corso di studi di Matematica, Informatica, Scienze Naturali e Tecniche (MINT). L'analisi si concentra sia sui fattori di natura personale che di contesto ambientale. I risultati mostrano che l'appartenenza all'uno o all'altro sesso, l'interesse per le materie matematico-scientifiche e i risultati ottenuti in queste discipline sono i fattori predittivi più attendibili rispetto ad una possibile scelta di studi MINT. Oltre a ciò, determinanti fondamentali per la scelta o l'esclusione di un corso di studi MINT sono le motivazioni intrinseche che spingono i/le ginnasiali verso un determinato percorso di studi.

Parole chiave: Ginnasio, scelta degli studi - MINT, sesso, motivi della scelta degli studi, interessi specifici per le materie 


\section{Study choice preferences of seconardy school students: Who decides to pursue a STEM degree and for what reasons?}

\section{Summary}

The article analyzes the study choice preferences of Swiss-German secondary school students. The central question focuses on the reasons why prospective students (de-)select a course of study in science, technology, engineering, and mathematics (STEM). We take into consideration both individual and contextual factors. The results show that the gender of the young people, as well as their interest and their achievement in mathematics and natural science subjects have the greatest predictive power for a STEM study choice. Moreover, the intrinsic study choice motives of high school students act as essential determinants of the selection or de-selection of a STEM degree.

Keywords: Secondary school, study choice, STEM, gender, study choice motives, subject-specific interests 